\title{
Existence of Periodic Solutions for Integrodifferential Impulsive Periodic System on Banach Space
}

\author{
JinRong Wang, ${ }^{1}$ X. Xiang, ${ }^{2}$ and W. Wei ${ }^{2}$ \\ ${ }^{1}$ College of Computer Science and Technology, Guizhou University, Guiyang, Guizhou 550025, China \\ ${ }^{2}$ College of Science, Guizhou University, Guiyang, Guizhou 550025, China
}

Correspondence should be addressed to JinRong Wang, wjr9668@126.com

Received 5 July 2008; Accepted 28 October 2008

Recommended by Agacik Zafer

This paper deals with a class of integrodifferential impulsive periodic systems on Banach space. Using impulsive periodic evolution operator given by us, the $T_{0}$-periodic $P C$-mild solution is introduced and suitable Poincaré operator is constructed. By virtue of the generalized new Gronwall lemma with impulse and B-norm, the estimate on the PC-mild solutions is derived. Showing the continuity and compactness of the Poincaré operator, we utilize Horn's fixed point theorem to prove the existence of $T_{0}$-periodic $P C$-mild solutions when the $P C$-mild solutions are bounded and ultimate bounded. This extends the study of periodic solutions of integrodifferential periodic system without impulse to integrodifferential periodic system with impulse on general Banach spaces. At last, an example is given for demonstration.

Copyright (C) 2008 JinRong Wang et al. This is an open access article distributed under the Creative Commons Attribution License, which permits unrestricted use, distribution, and reproduction in any medium, provided the original work is properly cited.

\section{Introduction}

It is well known that impulsive periodic motion is a very important and special phenomenon not only in natural science but also in social science such as climate, food supplement, insecticide population, and sustainable development. Periodic system with applications on finite dimensional spaces has been extensively studied. Particularly, impulsive periodic systems on finite dimensional spaces are considered and some important results (such as the existence and stability of periodic solution, the relationship between bounded solution and periodic solution, robustness by perturbation) are obtained (see [1-4]).

Since the end of the last century, many authors including us pay great attention to impulsive systems on infinite dimensional spaces. Ahmed investigated optimal control problems of a system governed by an impulsive system (see [5-8]). We also gave a series of results for semilinear (integrodifferential, strongly nonlinear) impulsive systems and 
optimal control problems (see [9-12]). Particulary, Benchohra et al. give many interesting recent results for various classes of differential equations with impulses on finite and infinite dimensional Banach spaces (see [13]).

Although, there are some papers on periodic solution for periodic system on infinite dimensional spaces (see [14-24]) and some results discussing integrodifferential system on finite Banach space and infinite Banach space (see $[19,25])$, to our knowledge, integrodifferential impulsive periodic systems on infinite dimensional spaces (with unbounded operator) have not been extensively investigated. Recently, we discuss the impulsive periodic system and integrodifferential impulsive system on infinite dimensional spaces. For linear impulsive evolution operator is constructed and $T_{0}$-periodic $P C$-mild solution is introduced. Existence of periodic solutions and alternative theorem, criteria of Massera type, and asymptotical stability and robustness by perturbation are established (see [26-28]). For integrodifferential impulsive system, existence of $P C$-mild solutions and optimal controls are presented (see [9]).

Herein, we go on studying the following integrodifferential impulsive periodic system:

$$
\begin{gathered}
\dot{x}(t)=A x(t)+f\left(t, x, \int_{0}^{t} g(t, s, x) d s\right), \quad t \neq \tau_{k}, \\
\Delta x(t)=B_{k} x(t)+c_{k}, \quad t=\tau_{k},
\end{gathered}
$$

on infinite dimensional Banach space $X$, where $0=\tau_{0}<\tau_{1}<\tau_{2}<\cdots<\tau_{k} \cdots, \lim _{k \rightarrow \infty} \tau_{k}=\infty$, $\tau_{k+\delta}=\tau_{k}+T_{0}, \Delta x\left(\tau_{k}\right)=x\left(\tau_{k}^{+}\right)-x\left(\tau_{k}^{-}\right), k \in \mathbb{Z}_{0}^{+}, T_{0}$ is a fixed positive number, and $\delta \in \mathbb{N}$ denotes the number of impulsive points between 0 and $T_{0}$. The operator $A$ is the infinitesimal generator of a $C_{0}$-semigroup $\{T(t), t \geq 0\}$ on $X, f$ is a measurable function from $[0, \infty) \times X \times X$ to $X$ and is $T_{0}$-periodic in $t, g$ is a continuous function from $[0, \infty) \times[0, \infty) \times X$ to $X$ and is $T_{0}$-periodic in $t$ and $s$ and $B_{k+\delta}=B_{k}, c_{k+\delta}=c_{k}$. This paper is mainly concerned with the existence of periodic solutions for integrodifferential impulsive periodic system on infinite dimensional Banach space $X$.

In this paper, we use Horn's fixed point theorem to obtain the existence of periodic solutions for integrodifferential impulsive periodic system (1.1). First, by virtue of impulsive evolution operator corresponding to linear homogeneous impulsive system, we construct a new Poincaré operator $P$ for integrodifferential impulsive periodic system (1.1), then overcome some difficulties to show the continuity and compactness of Poincaré operator $P$ which are very important. By a new generalized Gronwall inequality with impulse and $B$ norm given by us, the estimate of $P C$-mild solutions is established. Therefore, the existence of $T_{0}$-periodic $P C$-mild solutions for impulsive integrodifferential periodic system is shown. This extends the study of periodic solutions of integrodifferential periodic system without impulse to integrodifferential periodic system with impulse on general Banach spaces.

This paper is organized as follows. In Section 2, some results of linear impulsive periodic system and properties of impulsive periodic evolution operator corresponding to homogeneous linear impulsive periodic system are recalled. In Section 3, the new generalized Gronwall inequality with impulse and $B$-norm is established and the existence of $P C$-mild solutions for integrodifferential impulsive system is presented. In Section 4 , the $T_{0}$-periodic $P C$-mild solutions for integrodifferential impulsive periodic system (1.1) are introduced. We construct the suitable Poincaré operator $P$ and give the relation between $T_{0}$-periodic $P C$-mild solution and the fixed point of the Poincaré operator $P$. After showing the continuity and 
compactness of the Poincaré operator $P$, we can use Horn's fixed point theorem to establish the existence of $T_{0}$-periodic $P C$-mild solutions for integrodifferential impulsive periodic system when the $P C$-mild solutions are bounded and ultimate bounded. At last, an example is given to demonstrate the applicability of our result.

\section{Preliminaries}

In order to study the integrodifferential impulsive periodic system, we first recall some results about linear impulsive periodic system here. Let $X$ be a Banach space. $£(X)$ denotes the space of linear operators in $X$, and $£_{b}(X)$ denotes the space of bounded linear operators in $X$. $£_{b}(X)$ is the Banach space with the usual supremum norm. Define $\tilde{D}=\left\{\tau_{1}, \ldots, \tau_{\delta}\right\} \subset\left[0, T_{0}\right]$, where $\delta \in \mathbb{N}$ denotes the number of impulsive points between 0 and $T_{0}$. We introduce $P C\left(\left[0, T_{0}\right] ; X\right) \equiv\left\{x:\left[0, T_{0}\right] \rightarrow X \mid x\right.$ is continuous at $t \in\left[0, T_{0}\right] \backslash \widetilde{D}, x$ is continuous from left and has right-hand limits at $t \in \widetilde{D}\}$ and $P C^{1}\left(\left[0, T_{0}\right] ; X\right) \equiv\left\{x \in P C\left(\left[0, T_{0}\right] ; X\right) \mid \dot{x} \in\right.$ $\left.P C\left(\left[0, T_{0}\right] ; X\right)\right\}$. Set

$$
\|x\|_{P C}=\max \left\{\sup _{t \in\left[0, T_{0}\right]}\|x(t+0)\|, \sup _{t \in\left[0, T_{0}\right]}\|x(t-0)\|\right\}, \quad\|x\|_{P C^{1}}=\|x\|_{P C}+\|\dot{x}\|_{P C}
$$

It can be seen that a Banach space is endowed with the norm $\|\cdot\|_{P C}\left(\|\cdot\|_{P C^{1}}\right), P C\left(\left[0, T_{0}\right] ; X\right)$ $\left(P C^{1}\left(\left[0, T_{0}\right] ; X\right)\right)$.

Firstly, we consider the homogeneous linear impulsive periodic system

$$
\begin{aligned}
\dot{x}(t) & =A x(t), \quad t \neq \tau_{k}, \\
\Delta x(t) & =B_{k} x(t), \quad t=\tau_{k} .
\end{aligned}
$$

We introduce the following assumption.

\section{Assumption [H1].}

[H1.1] $A$ is the infinitesimal generator of a $C_{0}$-semigroup $\{T(t), t \geq 0\}$ on $X$ with domain $D(A)$.

[H1.2] There exists $\delta \in N$ such that $\tau_{k+\delta}=\tau_{k}+T_{0}$.

[H1.3] For each $k \in \mathbb{Z}_{0}^{+}, \delta \in N, B_{k} \in £_{b}(X)$ and $B_{k+\delta}=B_{k}$.

In order to study system (2.2), we need to consider the associated Cauchy problem

$$
\begin{gathered}
\dot{x}(t)=A x(t), \quad t \in\left[0, T_{0}\right] \backslash \tilde{D}, \\
\Delta x\left(\tau_{k}\right)=B_{k} x\left(\tau_{k}\right), \quad k=1,2, \ldots, \delta, \\
x(0)=\bar{x} .
\end{gathered}
$$

If $\bar{x} \in D(A)$ and $D(A)$ is an invariant subspace of $B_{k}$, using [29, Theorem 5.2.2, page $144]$, step by step, one can verify that the Cauchy problem (2.3) has a unique classical solution $x \in P C^{1}\left(\left[0, T_{0}\right] ; X\right)$ represented by $x(t)=S(t, 0) \bar{x}$ where

$$
S(\cdot, \cdot): \quad \Delta=\left\{(t, \theta) \in\left[0, T_{0}\right] \times\left[0, T_{0}\right] \mid 0 \leq \theta \leq t \leq T_{0}\right\} \longrightarrow £_{b}(X)
$$


given by

$$
S(t, \theta)= \begin{cases}T(t-\theta), & \tau_{k-1} \leq \theta \leq t \leq \tau_{k}, \\ T\left(t-\tau_{k}^{+}\right)\left(I+B_{k}\right) T\left(\tau_{k}-\theta\right), & \tau_{k-1} \leq \theta<\tau_{k}<t \leq \tau_{k+1}, \\ T\left(t-\tau_{k}^{+}\right)\left[\prod_{\theta<\tau_{j}<t}\left(I+B_{j}\right) T\left(\tau_{j}-\tau_{j-1}^{+}\right)\right]\left(I+B_{i}\right) T\left(\tau_{i}-\theta\right), \\ \tau_{i-1} \leq \theta<\tau_{i} \leq \cdots<\tau_{k}<t \leq \tau_{k+1} .\end{cases}
$$

The operator $\{S(t, \theta),(t, \theta) \in \Delta\}$ is called impulsive evolution operator associated with $\left\{B_{k} ; \tau_{k}\right\}_{k=1}^{\infty}$.

Now we introduce the $P C$-mild solution of Cauchy problem (2.3) and $T_{0}$-periodic $P C$ mild solution of the system (2.2).

Definition 2.1. For every $\bar{x} \in X$, the function $x \in P C\left(\left[0, T_{0}\right] ; X\right)$ given by $x(t)=S(t, 0) \bar{x}$ is said to be the $P C$-mild solution of the Cauchy problem (2.3).

Definition 2.2. A function $x \in P C([0,+\infty) ; X)$ is said to be a $T_{0}$-periodic $P C$-mild solution of system (2.2) if it is a $P C$-mild solution of Cauchy problem (2.3) corresponding to some $\bar{x}$ and $x\left(t+T_{0}\right)=x(t)$ for $t \geq 0$.

The following lemma gives the properties of the impulsive evolution operator $\{S(t, \theta),(t, \theta) \in \Delta\}$ associated with $\left\{B_{k} ; \tau_{k}\right\}_{k=1}^{\infty}$ which are widely used in the sequel.

Lemma 2.3 (see [26, Lemma 1]). Impulsive evolution operator $\{S(t, \theta),(t, \theta) \in \Delta\}$ has the following properties.

(1) For $0 \leq \theta \leq t \leq T_{0}, S(t, \theta) \in £_{b}(X)$, that is, $\sup _{0 \leq \theta \leq t \leq T_{0}}\|S(t, \theta)\| \leq M_{T_{0}}$, where $M_{T_{0}}>0$.

(2) For $0 \leq \theta<r<t \leq T_{0}, r \neq \tau_{k}, S(t, \theta)=S(t, r) S(r, \theta)$.

(3) For $0 \leq \theta \leq t \leq T_{0}$ and $N \in Z_{0}^{+}, S\left(t+N T_{0}, \theta+N T_{0}\right)=S(t, \theta)$.

(4) For $0 \leq t \leq T_{0}$ and $N \in Z_{0}^{+}, S\left(N T_{0}+t, 0\right)=S(t, 0)\left[S\left(T_{0}, 0\right)\right]^{N}$.

(5) If $\{T(t), t>0\}$ is a compact semigroup in $X$, then $S(t, \theta)$ is a compact operator for $0 \leq \theta<$ $t \leq T_{0}$.

Here, we note that system (2.2) has a $T_{0}$-periodic $P C$-mild solution $x$ if and only if $S\left(T_{0}, 0\right)$ has a fixed point. The impulsive evolution operator $\{S(t, \theta),(t, \theta) \in \Delta\}$ can be used to reduce the existence of $T_{0}$-periodic $P C$-mild solutions for linear impulsive periodic system to the existence of fixed points for an operator equation. This implies that we can build up the new framework to study the periodic $P C$-mild solutions for integrodifferential impulsive periodic system on Banach space.

Consider the nonhomogeneous linear impulsive periodic system

$$
\begin{array}{cl}
\dot{x}(t)=A x(t)+f(t), & t \neq \tau_{k}, \\
\Delta x(t)=B_{k} x(t)+c_{k}, & t=\tau_{k},
\end{array}
$$


and the associated Cauchy problem

$$
\begin{gathered}
\dot{x}(t)=A x(t)+f(t), \quad t \in\left[0, T_{0}\right] \backslash \tilde{D}, \\
\Delta x\left(\tau_{k}\right)=B_{k} x\left(\tau_{k}\right)+c_{k}, \quad k=1,2, \ldots, \delta, \\
x(0)=\bar{x},
\end{gathered}
$$

where $f \in L^{1}\left(\left[0, T_{0}\right] ; X\right), f\left(t+T_{0}\right)=f(t)$, and $c_{k+\delta}=c_{k}$.

Now we introduce the $P C$-mild solution of Cauchy problem (2.7) and $T_{0}$-periodic $P C$ mild solution of system (2.6).

Definition 2.4. A function $x \in P C\left(\left[0, T_{0}\right] ; X\right)$, for finite interval $\left[0, T_{0}\right]$, is said to be a $P C$-mild solution of the Cauchy problem (2.6) corresponding to the initial value $\bar{x} \in X$ and input $f \in L^{1}\left(\left[0, T_{0}\right] ; X\right)$ if $x$ is given by

$$
x(t)=S(t, 0) \bar{x}+\int_{0}^{t} S(t, \theta) f(\theta) d \theta+\sum_{0 \leq \tau_{k}<t} S\left(t, \tau_{k}^{+}\right) c_{k} .
$$

Definition 2.5. A function $x \in P C([0,+\infty) ; X)$ is said to be a $T_{0}$-periodic $P C$-mild solution of system (2.6) if it is a PC-mild solution of Cauchy problem (2.7) corresponding to some $\bar{x}$ and $x\left(t+T_{0}\right)=x(t)$ for $t \geq 0$.

\section{The generalized Gronwall inequality and existence of solutions}

In order to derive an estimate of the $P C$-mild solutions, we introduce the following generalized Gronwall inequality with impulse and $B$-norm.

Lemma 3.1. Let $x \in P C([0, \infty), X)$ and let it satisfy the following inequality:

$$
\|x(t)\| \leq a+b \int_{0}^{t}\|x(\theta)\|^{\lambda_{1}} d \theta+c \int_{0}^{t}\left\|x_{\theta}\right\|_{B}^{\lambda_{2}} d \theta,
$$

where $a, b, c \geq 0,0 \leq \lambda_{1}, \lambda_{2} \leq 1$ are constants, and

$$
\left\|x_{\theta}\right\|_{B}=\max \left\{\sup _{0 \leq \xi \leq \theta}\|x(\xi+0)\|, \sup _{0 \leq \xi \leq \theta}\|x(\xi-0)\|\right\} .
$$

Then one has

$$
\|x(t)\| \leq(a+1) e^{(b+c) t}
$$

Proof. (i) For $0 \leq \lambda_{1}, \lambda_{2}<1$, let $\lambda=\max \left\{\lambda_{1}, \lambda_{2}\right\} \in[0,1)$ and

$$
y(t)= \begin{cases}1, & \|x(t)\| \leq 1 \\ x(t), & \|x(t)\|>1\end{cases}
$$


Then

$$
\|x(t)\| \leq\|y(t)\| \leq(a+1)+b \int_{0}^{t}\|y(\theta)\|^{\lambda} d \theta+c \int_{0}^{t}\left\|y_{\theta}\right\|_{B}^{\lambda} d \theta, \quad \forall t \in\left[0, T_{0}\right]
$$

Using (3.5), we obtain

$$
\left\|y_{t}\right\|_{B}^{\lambda} \leq(a+1)+(b+c) \int_{0}^{t}\left\|y_{\theta}\right\|_{B}^{\lambda} d \theta
$$

Define

$$
u(t)=(a+1)+(b+c) \int_{0}^{t}\left\|y_{\theta}\right\|_{B}^{\lambda} d \theta
$$

then we get

$$
\begin{gathered}
\dot{u}(t)=(b+c)\left\|y_{t}\right\|_{B^{\prime}}^{\lambda} \quad t \neq \tau_{k} \\
u(0)=a+1, \quad u\left(\tau_{k}+0\right)=u\left(\tau_{k}\right) .
\end{gathered}
$$

Since $\left\|y_{t}\right\|_{B}^{\lambda} \leq u(t)$, we then have

$$
\begin{gathered}
\dot{u}(t) \leq(b+c) u(t), \quad t \neq \tau_{k}, \\
u(0)=a+1, \quad u\left(\tau_{k}+0\right)=u\left(\tau_{k}\right) .
\end{gathered}
$$

For $t \in\left(\tau_{k}, \tau_{k+1}\right]$, by (3.9), we obtain

$$
u(t) \leq u\left(\tau_{k}+0\right) e^{(b+c)\left(t-\tau_{k}\right)}=u\left(\tau_{k}\right) e^{(b+c)\left(t-\tau_{k}\right)}
$$

further,

$$
u(t) \leq(a+1) e^{(b+c) t}
$$

thus,

$$
\|x(t)\| \leq\left\|y_{t}\right\|_{B} \leq(a+1) e^{(b+c) t}
$$

(ii) For $\lambda_{1}=\lambda_{2}=1$, we only need to define

$$
u_{1}(t)=a+(b+c) \int_{0}^{t}\left\|x_{\theta}\right\|_{B} d \theta
$$


Similar to the proof in (i), one can obtain

$$
\|x(t)\| \leq\left\|x_{t}\right\|_{B} \leq a e^{(b+c) t} .
$$

Combining (i) and (ii), one can complete the proof.

Now, we consider the following integrodifferential impulsive periodic system:

$$
\begin{gathered}
\dot{x}(t)=A x(t)+f\left(t, x, \int_{0}^{t} g(t, s, x) d s\right), \quad t \neq \tau_{k}, \\
\Delta x(t)=B_{k} x(t)+c_{k}, \quad t=\tau_{k},
\end{gathered}
$$

and the associated Cauchy problem

$$
\begin{gathered}
\dot{x}(t)=A x(t)+f\left(t, x, \int_{0}^{t} g(t, s, x) d s\right), \quad t \in\left[0, T_{0}\right] \backslash \tilde{D}, \\
\Delta x\left(\tau_{k}\right)=B_{k} x\left(\tau_{k}\right)+c_{k}, \quad k=1,2, \ldots, \delta, \\
x(0)=\bar{x} .
\end{gathered}
$$

By virtue of the expression of the $P C$-mild solution of the Cauchy problem (2.7), we can introduce the $P C$-mild solution of the Cauchy problem (3.16).

Definition 3.2. A function $x \in P C\left(\left[0, T_{0}\right] ; X\right)$ is said to be a $P C$-mild solution of the Cauchy problem (3.16) corresponding to the initial value $\bar{x} \in \mathrm{X}$ if $x$ satisfies the following integral equation:

$$
\begin{aligned}
x(t)= & S(t, 0) \bar{x}+\int_{0}^{t} S(t, \theta) f\left(\theta, x(\theta), \int_{0}^{\theta} g(\theta, s, x(s)) d s\right) d \theta \\
& +\sum_{0 \leq \tau_{k}<t} S\left(t, \tau_{k}^{+}\right) c_{k}, \quad \text { for } t \in\left[0, T_{0}\right] .
\end{aligned}
$$

Now, we introduce the $T_{0}$-periodic $P C$-mild solution of system (3.15).

Definition 3.3. A function $x \in P C([0,+\infty) ; X)$ is said to be a $T_{0}$-periodic $P C$-mild solution of system (3.15) if it is a $P C$-mild solution of Cauchy problem (3.16) corresponding to some $\bar{x}$ and $x\left(t+T_{0}\right)=x(t)$ for $t \geq 0$.

\section{Assumption [H2].}

[H2.1] $f:[0, \infty) \times X \times X \rightarrow X$ is measurable for $t \geq 0$, and for any $x_{1}, x_{2}, y_{1}, y_{2} \in X$ satisfying $\left\|x_{1}\right\|,\left\|x_{2}\right\|,\left\|y_{1}\right\|,\left\|y_{2}\right\| \leq \rho$ there exists a positive constant $L_{f}(\rho)>0$ such that

$$
\left\|f\left(t, x_{1}, y_{1}\right)-f\left(t, x_{2}, y_{2}\right)\right\| \leq L_{f}(\rho)\left(\left\|x_{1}-x_{2}\right\|+\left\|y_{1}-y_{2}\right\|\right)
$$


[H2.2] There exists a positive constant $M_{f}>0$ such that

$$
\|f(t, x, y)\| \leq M_{f}(1+\|x\|+\|y\|), \quad \forall x, y \in X
$$

[H2.3] $f(t, x, y)$ is $T_{0}$-periodic in $t$, that is,

$$
f\left(t+T_{0}, x, y\right)=f(t, x, y), \quad t \geq 0 .
$$

[H2.4] $g:[0, \infty) \times[0, \infty) \times X \rightarrow X$ is continuous for $t \geq s \geq 0$ and for any $x, y \in X$ satisfying $\|x\|,\|y\| \leq \rho$ there exists a positive constant $L_{g}(\rho)>0$ such that

$$
\|g(t, s, x)-g(t, s, y)\| \leq L_{g}(\rho)\|x-y\|
$$

[H2.5] There exists a positive constant $M_{g}>0$ such that

$$
\|g(t, s, x)\| \leq M_{g}(1+\|x\|), \quad \forall x \in X
$$

[H2.6] $g(t, s, x)$ are $T_{0}$-periodic in $t$ and $s$, that is,

$$
\begin{gathered}
g\left(t+T_{0}, s+T_{0}, x\right)=g(t, s, x), \quad t \geq s \geq 0, \\
\int_{0}^{T_{0}} g(t, s, x) d s=0, \quad t \geq s \geq 0 .
\end{gathered}
$$

[H2.7] For each $k \in \mathbb{Z}_{0}^{+}$and $c_{k} \in X$ there exists $\delta \in \mathbb{N}$ such that $c_{k+\delta}=c_{k}$.

It is not difficult to verify the following results.

Lemma 3.4. Under the assumptions [H2.4] and [H2.5], one has the following properties:

(1) $\int_{0} g(\cdot, s, x(s)) d s: P C\left(\left[0, T_{0}\right] ; X\right) \rightarrow P C\left(\left[0, T_{0}\right] ; X\right)$;

(2) for all $x_{1}, x_{2} \in P C\left(\left[0, T_{0}\right] ; X\right)$ and $\left\|x_{1}\right\|_{P C\left(\left[0, T_{0}\right] ; X\right)},\left\|x_{2}\right\|_{P C\left(\left[0, T_{0}\right] ; X\right)} \leq \rho$,

$$
\left\|\int_{0}^{t} g\left(t, s, x_{1}(s)\right) d s-\int_{0}^{t} g\left(t, s, x_{2}(s)\right) d s\right\| \leq L_{g}(\rho) T_{0}\left\|\left(x_{1}\right)_{t}-\left(x_{2}\right)_{t}\right\|_{B^{\prime}}
$$

(3) for $x \in P C\left(\left[0, T_{0}\right] ; X\right)$,

$$
\left\|\int_{0}^{t} g(t, s, x(s)) d s\right\| \leq M_{g} T_{0}\left(1+\left\|x_{t}\right\|_{B}\right)
$$

Now we present the existence of $P C$-mild solution for system (3.16). 
Theorem 3.5. Assumptions [H1.1], [H2.1], [H2.2], [H2.4], and [H2.5] hold, and for each $k \in \mathbb{Z}_{0}^{+}$, $B_{k} \in £_{b}(X), c_{k} \in$ Xare fixed. Then system (3.16) has a unique PC-mild solution given by

$$
x(t, \bar{x})=S(t, 0) \bar{x}+\int_{0}^{t} S(t, \theta) f\left(\theta, x(\theta), \int_{0}^{\theta} g(\theta, s, x(s)) d s\right) d \theta+\sum_{0 \leq \tau_{k}<t} S\left(t, \tau_{k}^{+}\right) c_{k} .
$$

Proof. A similar result is given by Wei et al. in [9]. Thus, we only sketch the proof here. In order to make the process clear we divide it into three steps.

Step 1. We consider the following general integrodifferential equation without impulse

$$
\begin{gathered}
\dot{x}(t)=A x(t)+f\left(t, x, \int_{0}^{t} g(t, s, x) d s\right), \quad t \in[s, \tau], \\
x(s)=\bar{x} \in X .
\end{gathered}
$$

In order to obtain the local existence of mild solution for system (3.27), we only need to set up the framework for using the contraction mapping theorem. Consider the ball given by

$$
\mathfrak{B}=\left\{x \in C\left(\left[s, t_{1}\right] ; X\right) \mid\|x(t)-\bar{x}\| \leq 1, s \leq t \leq t_{1}\right\},
$$

where $t_{1}$ would be chosen and $\|x(t)\| \leq 1+\|\bar{x}\|=\bar{\rho}, s \leq t \leq t_{1} \cdot \mathfrak{B} \subseteq C\left(\left[s, t_{1}\right], X\right)$ is a closed convex set. Define a map $\mathbf{Q}$ on $\mathfrak{B}$ given by

$$
(\mathbf{Q} x)(t)=T(t) \bar{x}+\int_{s}^{t} T(t-\theta) f\left(\theta, x(\theta), \int_{0}^{\theta} g(\theta, s, x(s)) d s\right) d \theta .
$$

Under the assumptions [H1.1], [H2.1], [H2.2], [H2.4], [H2.5], and Lemma 3.1, one can verify that map $\mathbf{Q}$ is a contraction map on $\mathfrak{B}$ with chosen $t_{1}>0$. This means that system (3.27) has a unique mild solution $x \in C\left(\left[s, t_{1}\right] ; X\right)$ given by

$$
x(t)=T(t) \bar{x}+\int_{s}^{t} T(t-\theta) f\left(\theta, x(\theta), \int_{0}^{\theta} g(\theta, s, x(s)) d s\right) d \theta \quad \text { on }\left[s, t_{1}\right]
$$

Again, using Lemma 3.1, we can obtain the a priori estimate of the mild solutions for system (3.27) and present the global existence of mild solutions. 
Step 2. For $t \in\left(\tau_{k}, \tau_{k+1}\right]$, consider the Cauchy problem

$$
\begin{gathered}
\dot{x}(t)=A x(t)+f\left(t, x, \int_{0}^{t} g(t, s, x) d s\right), \quad t \in\left(\tau_{k}, \tau_{k+1}\right], \\
x\left(\tau_{k}\right)=x_{k} \equiv\left(I+B_{k}\right) x\left(\tau_{k}\right)+c_{k} \in X .
\end{gathered}
$$

By Step 1, Cauchy problem (3.31) also has a unique PC-mild solution:

$$
x(t)=T\left(t-\tau_{k}\right) x_{k}+\int_{\tau_{k}}^{t} T(t-\theta) f\left(\theta, x(\theta), \int_{0}^{\theta} g(\theta, s, x(s)) d s\right) d \theta .
$$

Step 3. Combining all solutions on $\left(\tau_{k}, \tau_{k+1}\right](k=1, \ldots, \delta)$, one can obtain the PC-mild solution of the Cauchy problem (3.16) given by

$$
x(t, \bar{x})=S(t, 0) \bar{x}+\int_{0}^{t} S(t, \theta) f\left(\theta, x(\theta), \int_{0}^{\theta} g(\theta, s, x(s)) d s\right) d \theta+\sum_{0 \leq \tau_{k}<t} S\left(t, \tau_{k}^{+}\right) c_{k} .
$$

This completes the proof.

\section{Poincaré operator and existence of periodic solutions}

To establish the periodic solutions for the system (3.15), we define a Poincaré operator from $X$ to $X$ as follows:

$$
\begin{aligned}
P(\bar{x}) & =x\left(T_{0}, \bar{x}\right) \\
& =S\left(T_{0}, 0\right) \bar{x}+\int_{0}^{T_{0}} S\left(T_{0}, \theta\right) f\left(\theta, x(\theta, \bar{x}), \int_{0}^{\theta} g(\theta, s, x(s, \bar{x})) d s\right) d \theta+\sum_{0 \leq \tau_{k}<T_{0}} S\left(T_{0}, \tau_{k}^{+}\right) c_{k},
\end{aligned}
$$

where $x(\cdot, \bar{x})$ denotes the $P C$-mild solution of the Cauchy problem (3.16) corresponding to the initial value $x(0)=\bar{x}$, then examine whether $P$ has a fixed point.

We first note that a fixed point of the Poincaré operator $P$ gives rise to a periodic solution.

Lemma 4.1. System (3.15) has a $T_{0}$-periodic PC-mild solution if and only if $P$ has a fixed point.

Proof. Suppose $x(\cdot)=x\left(\cdot+T_{0}\right)$, then $x(0)=x\left(T_{0}\right)=P(x(0))$. This implies that $x(0)$ is a fixed point of $P$. On the other hand, if $P x_{0}=x_{0}, x_{0} \in X$, then for the $P C$-mild solution $x\left(\cdot, x_{0}\right)$ of the Cauchy problem (3.16) corresponding to the initial value $x(0)=x_{0}$, we can define $y(\cdot)=x\left(\cdot+T_{0}, x_{0}\right)$, then $y(0)=x\left(T_{0}, x_{0}\right)=P x_{0}=x_{0}$. Now, for $t>0$, we can use the properties 
JinRong Wang et al.

(2), (3), and (4) of Lemma 2.3 and assumptions [H1.2], [H1.3], [H2.3], [H2.6], and [H2.7] to arrive at

$$
\begin{aligned}
& y(t)=x\left(t+T_{0}, x_{0}\right) \\
& =S\left(t+T_{0}, T_{0}\right) S\left(T_{0}, 0\right) x_{0} \\
& +\int_{0}^{T_{0}} S\left(t+T_{0}, T_{0}\right) S\left(T_{0}, \theta\right) f\left(\theta, x\left(\theta, x_{0}\right), \int_{0}^{\theta} g\left(\theta, s, x\left(s, x_{0}\right)\right) d s\right) d \theta \\
& +\sum_{0 \leq \tau_{k}<T_{0}} S\left(t+T_{0}, T_{0}\right) S\left(T_{0}, \tau_{k}^{+}\right) c_{k} \\
& +\int_{T_{0}}^{t+T_{0}} S\left(t+T_{0}, \theta\right) f\left(\theta, x\left(\theta, x_{0}\right), \int_{0}^{\theta} g\left(\theta, s, x\left(s, x_{0}\right)\right) d s\right) d \theta \\
& +\sum_{T_{0} \leq \tau_{k+\delta}<t+T_{0}} S\left(t+T_{0}, \tau_{k+\delta}^{+}\right) c_{k+\delta} \\
& =S(t, 0)\left\{S\left(T_{0}, 0\right) x_{0}+\int_{0}^{T_{0}} S\left(T_{0}, \theta\right) f\left(\theta, x\left(\theta, x_{0}\right), \int_{0}^{\theta} g\left(\theta, s, x\left(s, x_{0}\right)\right) d s\right) d \theta\right. \\
& \left.+\sum_{0 \leq \tau_{k}<T_{0}} S\left(T_{0}, \tau_{k}^{+}\right) c_{k+\delta}\right\} \\
& +\int_{0}^{t} S\left(t+T_{0}, \theta+T_{0}\right) f\left(\theta+T_{0}, x\left(\theta+T_{0}, x_{0}\right), \int_{0}^{\theta+T_{0}} g\left(\theta+T_{0}, s, x\left(s, x_{0}\right)\right) d s\right) d \theta \\
& +\sum_{T_{0} \leq \tau_{k+\delta}<t+T_{0}} S\left(t+T_{0}, \tau_{k+\delta}^{+}\right) c_{k+\delta} \\
& =S(t, 0) x\left(T_{0}\right) \\
& +\int_{0}^{t} S\left(t+T_{0}, \theta+T_{0}\right) f\left(\theta+T_{0}, x\left(\theta+T_{0}, x_{0}\right), \int_{T_{0}}^{\theta+T_{0}} g\left(\theta+T_{0}, s, x\left(s, x_{0}\right)\right) d s\right) d \theta \\
& +\sum_{T_{0} \leq \tau_{k+\delta}<t+T_{0}} S\left(t+T_{0}, \tau_{k+\delta}^{+}\right) c_{k+\delta} \\
& =S(t, 0) x\left(T_{0}\right) \\
& +\int_{0}^{t} S(t, \theta) f\left(\theta, x\left(\theta+T_{0}, x_{0}\right), \int_{0}^{\theta} g\left(\theta+T_{0}, s+T_{0}, x\left(s+T_{0}, x_{0}\right)\right) d s\right) d \theta \\
& +\sum_{T_{0} \leq \tau_{k+\delta}<t+T_{0}} S\left(t+T_{0}, \tau_{k+\delta}^{+}\right) c_{k+\delta} \\
& =S(t, 0) y(0)+\int_{0}^{t} S(t, \theta) f\left(\theta, y(\theta, y(0)), \int_{0}^{\theta} g(\theta, s, y(s, y(0))) d s\right) d \theta \\
& +\sum_{0 \leq \tau_{k}<t} S\left(t, \tau_{k}^{+}\right) c_{k} \\
& =S(t, 0) y(0)+\int_{0}^{t} S(t, \theta) f\left(\theta, y(\theta, y(0)), \int_{0}^{\theta} g(\theta, s, y(s, y(0))) d s\right) d \theta \\
& +\sum_{0 \leq \tau_{k}<t} S\left(t, \tau_{k}^{+}\right) c_{k}
\end{aligned}
$$


This implies that $y(\cdot, y(0))$ is a $P C$-mild solution of Cauchy problem (3.16) with initial value $y(0)=x_{0}$. Thus the uniqueness implies that $x\left(\cdot, x_{0}\right)=y(\cdot, y(0))=x\left(\cdot+T_{0}, x_{0}\right)$, so that $x\left(\cdot, x_{0}\right)$ is a $T_{0}$-periodic.

Second, we show that the Poincaré operator $P$ defined by (4.1) is a continuous operator.

Lemma 4.2. Assumptions [H1.1], [H2.1], [H2.2], [H2.4], and [H2.5] hold. Then the operator $P$ is a continuous operator of $\bar{x}$ on $\mathrm{X}$.

Proof. Let $\bar{x}, \bar{y} \in \Xi \subset X$, where $\Xi$ is a bounded subset of $X$. Suppose $x(\cdot, \bar{x})$ and $x(\cdot, \bar{y})$ are the $P C$-mild solutions of Cauchy problem (3.16) corresponding to the initial value $\bar{x}$ and $\bar{y} \in X$, respectively, given by

$$
\begin{aligned}
& x(t, \bar{x})=S(t, 0) \bar{x}+\int_{0}^{t} S(t, \theta) f\left(\theta, x(\theta, \bar{x}), \int_{0}^{\theta} g(\theta, s, x(s, \bar{x})) d s\right) d \theta+\sum_{0 \leq \tau_{k}<t} S\left(T_{0}, \tau_{k}^{+}\right) c_{k} ; \\
& x(t, \bar{y})=S(t, 0) \bar{y}+\int_{0}^{t} S(t, \theta) f\left(\theta, x(\theta, \bar{y}), \int_{0}^{\theta} g(\theta, s, x(s, \bar{y})) d s\right) d \theta+\sum_{0 \leq \tau_{k}<t} S\left(T_{0}, \tau_{k}^{+}\right) c_{k} .
\end{aligned}
$$

Thus, by assumption [H2.2], property (1) of Lemma 2.3, and property (3) of Lemma 3.4, we obtain

$$
\begin{aligned}
\|x(t, \bar{x})\| \leq & M_{T_{0}}\|\bar{x}\|+\left(1+M_{g} T_{0}\right) M_{T_{0}} M_{f} T_{0}+M_{T_{0}} \sum_{0 \leq \tau_{k}<T_{0}}\left\|c_{k}\right\| \\
& +M_{T_{0}} M_{f} \int_{0}^{t}\|x(\theta, \bar{x})\| d \theta+M_{T_{0}} M_{f} M_{g} T_{0} \int_{0}^{t}\left\|x_{\theta, \bar{x}}\right\|_{B} d \theta \\
\leq & a_{0}+M_{T_{0}} M_{f} \int_{0}^{t}\|x(\theta, \bar{x})\| d \theta+M_{T_{0}} M_{f} M_{g} T_{0} \int_{0}^{t}\left\|x_{\theta, \bar{x}}\right\|_{B} d \theta \\
\|x(t, \bar{y})\| \leq & M_{T_{0}}\|\bar{y}\|+\left(1+M_{g} T_{0}\right) M_{T_{0}} M_{f} T_{0}+M_{T_{0}} \sum_{0 \leq \tau_{k}<T_{0}}\left\|c_{k}\right\| \\
& +M_{T_{0}} M_{f} \int_{0}^{t}\|x(\theta, \bar{y})\| d \theta+M_{T_{0}} M_{f} M_{g} T_{0} \int_{0}^{t}\left\|x_{\theta, \bar{y}}\right\|_{B} d \theta \\
\leq & b_{0}+M_{T_{0}} M_{f} \int_{0}^{t}\|x(\theta, \bar{y})\| d \theta+M_{T_{0}} M_{f} M_{g} T_{0} \int_{0}^{t}\left\|x_{\theta, \bar{y}}\right\|_{B} d \theta .
\end{aligned}
$$

By Lemma 3.1, one can verify that there exist constants $M_{1}^{*}$ and $M_{2}^{*}>0$ such that

$$
\|x(t, \bar{x})\| \leq M_{1}^{*}, \quad\|x(t, \bar{y})\| \leq M_{2}^{*} .
$$

Let $\rho=\max \left\{M_{1}^{*}, M_{2}^{*}\right\}>0$, then $\|x(\cdot, \bar{x})\|,\|x(\cdot, \bar{y})\| \leq \rho$ which imply that they are locally bounded. 
By assumption [H2.1], property (1) of Lemma 2.3, and property (2) of Lemma 3.4, we obtain

$$
\begin{aligned}
& \|x(t, \bar{x})-x(t, \bar{y})\| \\
& \leq\|S(t, 0)\|\|\bar{x}-\bar{y}\| \\
& \quad+\int_{0}^{t}\|S(t, \theta)\| \| f\left(\theta, x(\theta, \bar{x}), \int_{0}^{\theta} g(\theta, s, x(s, \bar{x})) d s\right) \\
& \quad-f\left(\theta, x(\theta, \bar{y}), \int_{0}^{\theta} g(\theta, s, x(s, \bar{y})) d s\right) \| d \theta \\
& \leq M_{T_{0}}\|\bar{x}-\bar{y}\| \quad+M_{T_{0}} L_{f}(\rho) \int_{0}^{t}\|x(\theta, \bar{x})-x(\theta, \bar{y})\| d \theta+M_{T_{0}} L_{f}(\rho) L_{g}(\rho) T_{0} \int_{0}^{t}\left\|x_{\theta, \bar{x}}-x_{\theta, \bar{y}}\right\|_{B} d \theta .
\end{aligned}
$$

By Lemma 3.1 again, one can verify that there exists a constant $M_{3}^{*}>0$ such that

$$
\|x(t, \bar{x})-x(t, \bar{y})\| \leq M_{3}^{*} M_{T_{0}}\|\bar{x}-\bar{y}\| \equiv L\|\bar{x}-\bar{y}\|, \quad \forall t \in\left[0, T_{0}\right]
$$

which implies that

$$
\|P(\bar{x})-P(\bar{y})\|=\left\|x\left(T_{0}, \bar{x}\right)-x\left(T_{0}, \bar{y}\right)\right\| \leq L\|\bar{x}-\bar{y}\| .
$$

Hence, $P$ is a continuous operator of $\bar{x}$ on $X$.

In the sequel, we need to prove the compactness of operator $P$, so we make the following assumption.

Assumption [H3] The semigroup $\{T(t), t>0\}$ is a compact semigroup on $X$.

Now, we are ready to prove the compactness of operator $P$ defined by (4.1).

Lemma 4.3. Assumptions [H1.1], [H2.1], [H2.2], [H2.4], [H2.5], and [H3] hold. Then the operator $P$ is a compact operator.

Proof. We only need to verify that $P$ takes a bounded set into a precompact set in $X$.

Let $\Gamma$ be a bounded subset of $X$. Define $K=P \Gamma=\{P(\bar{x}) \in X \mid \bar{x} \in \Gamma\}$.

For $0<\varepsilon \leq T_{0}$, define

$$
K_{\varepsilon}=P_{\varepsilon} \Gamma=S\left(T_{0}, T_{0}-\varepsilon\right)\left\{x\left(T_{0}-\varepsilon, \bar{x}\right) \mid \bar{x} \in \Gamma\right\} .
$$


Next, we show that $K_{\varepsilon}$ is precompact in $X$. In fact, for $\bar{x} \in \Gamma$ fixed, we have

$$
\begin{aligned}
&\left\|x\left(T_{0}-\varepsilon, \bar{x}\right)\right\| \\
& \leq\left\|S\left(T_{0}-\varepsilon, 0\right) \bar{x}\right\|+\int_{0}^{T_{0}-\varepsilon}\left\|S\left(T_{0}-\varepsilon, \theta\right) f\left(\theta, x(\theta, \bar{x}), \int_{0}^{\theta} g(\theta, s, x(s, \bar{x})) d s\right)\right\| d \theta \\
&+\sum_{0 \leq \tau_{k}<T_{0}-\varepsilon}\left\|S\left(T_{0}-\varepsilon, \tau_{k}^{+}\right) c_{k}\right\| \\
& \leq M_{T_{0}}\|\bar{x}\|+M_{T_{0}} M_{f} T_{0}\left(1+M_{g} T_{0}\right)+M_{T_{0}} M_{f} \int_{0}^{T_{0}}\|x(\theta, \bar{x})\| d \theta \\
&+M_{T_{0}} \sum_{0 \leq \tau_{k}<T_{0}}\left\|c_{k}\right\|+M_{T_{0}} M_{f} M_{g} T_{0} \int_{0}^{T_{0}}\left\|x_{\theta, \bar{x}}\right\|_{B} d \theta \\
& \leq M_{T_{0}}\|\bar{x}\|+M_{T_{0}} M_{f} T_{0}\left(1+M_{g} T_{0}\right)+\left(1+M_{g} T_{0}\right) M_{T_{0}} M_{f} T_{0} \rho+M_{T_{0}} \sum_{0 \leq \tau_{k}<T_{0}}\left\|c_{k}\right\| .
\end{aligned}
$$

This implies that the set $\left\{x\left(T_{0}-\varepsilon, \bar{x}\right) \mid \bar{x} \in \Gamma\right\}$ is totally bounded.

By virtue of the compactness of $\{T(t), t>0\}$ and property (5) of Lemma 2.3, $S\left(T_{0}, T_{0}-\right.$ $\varepsilon)$ is a compact operator. Thus, $K_{\varepsilon}$ is precompact in $X$.

On the other hand, for arbitrary $\bar{x} \in \Gamma$,

$$
\begin{aligned}
P_{\varepsilon}(\bar{x})= & S\left(T_{0}, 0\right) \bar{x}+\int_{0}^{T_{0}-\varepsilon} S\left(T_{0}, \theta\right) f\left(\theta, x(\theta, \bar{x}), \int_{0}^{\theta} g(\theta, s, x(s, \bar{x})) d s\right) d \theta \\
& +\sum_{0 \leq \tau_{k}<T_{0}-\varepsilon} S\left(T_{0}, \tau_{k}^{+}\right) c_{k} .
\end{aligned}
$$

Thus, combined with (4.1), we have

$$
\begin{aligned}
\| P_{\varepsilon}(\bar{x})- & P(\bar{x}) \| \\
\leq & \| \int_{0}^{T_{0}-\varepsilon} S\left(T_{0}, \theta\right) f\left(\theta, x(\theta, \bar{x}), \int_{0}^{\theta} g(\theta, s, x(s, \bar{x})) d s\right) d \theta \\
& \quad-\int_{0}^{T_{0}} S\left(T_{0}, \theta\right) f\left(\theta, x(\theta, \bar{x}), \int_{0}^{\theta} g(\theta, s, x(s, \bar{x})) d s\right) d \theta \| \\
& +\left\|\sum_{0 \leq \tau_{k}<T_{0}-\varepsilon} S\left(T_{0}, \tau_{k}^{+}\right) c_{k}-\sum_{0 \leq \tau_{k}<T_{0}} S\left(T_{0}, \tau_{k}^{+}\right) c_{k}\right\| \\
\leq & \int_{T_{0}-\varepsilon}^{T_{0}}\left\|S\left(T_{0}, \theta\right)\right\|\left\|f\left(\theta, x(\theta, \bar{x}), \int_{0}^{\theta} g(\theta, s, x(s, \bar{x})) d s\right)\right\| d \theta+M_{T_{0}} \sum_{T_{0}-\varepsilon \leq \tau_{k}<T_{0}}\left\|c_{k}\right\| \\
\leq & M_{T_{0}} M_{f}\left(1+M_{g} T_{0}\right)(1+\rho) \varepsilon+M_{T_{0}} \sum_{T_{0}-\varepsilon \leq \tau_{k}<T_{0}}\left\|c_{k}\right\| .
\end{aligned}
$$


It is showing that the set $K$ can be approximated to an arbitrary degree of accuracy by a precompact set $K_{\varepsilon}$. Hence $K$ itself is a precompact set in $X$, that is, $P$ takes a bounded set into a precompact set in $X$. As a result, $P$ is a compact operator.

After showing the continuity and compactness of operator $P$, we can follow and derive periodic $P C$-mild solutions for system (3.15). In the sequel, we define the following definitions. The following definitions are standard, we state them here for convenient references. Note that the uniform boundedness and uniform ultimate boundedness are not required to obtain the periodic $P C$-mild solutions here, so we only define the (locally) boundedness and ultimate boundedness.

Definition 4.4. One says that $P C$-mild solutions of Cauchy problem (3.16) are bounded if for each $B_{1}>0$, there is a $B_{2}>0$ such that $\|\bar{x}\| \leq B_{1}$ implies $\|x(t, \bar{x})\| \leq B_{2}$ for $t \geq 0$.

Definition 4.5. One says that $P C$-mild solutions of Cauchy problem (3.16) are locally bounded if for each $B_{1}>0$ and $k_{0}>0$, there is a $B_{2}>0$ such that $\|\bar{x}\| \leq B_{1}$ implies $\|x(t, \bar{x})\| \leq B_{2}$ for $0 \leq t \leq k_{0}$.

Definition 4.6. One says that $P C$-mild solutions of Cauchy problem (3.16) are ultimate bounded if there is a bound $B>0$ such that for each $B_{3}>0$, there is a $k>0$, such that $\|\bar{x}\| \leq B_{3}$ and $t \geq k$ imply $\|x(t, \bar{x})\| \leq B$.

We also need the following results as a reference.

Lemma 4.7 (see [21, Theorem 3.1]). Local boundedness and ultimate boundedness imply boundedness and ultimate boundedness.

Lemma 4.8 (Horn's fixed point theorem [20, Lemma 3.1]). Let $E_{0} \subset E_{1} \subset E_{2}$ be convex subsets of Banach space $X$, with $E_{0}$ and $E_{2}$ compact subsets and $E_{1}$ open relative to $E_{2}$. Let $P: E_{2} \rightarrow X$ be a continuous map such that for some integer $m$, one has

$$
\begin{gathered}
P^{j}\left(E_{1}\right) \subset E_{2}, \quad 1 \leq j \leq m-1, \\
P^{j}\left(E_{1}\right) \subset E_{0}, \quad m \leq j \leq 2 m-1,
\end{gathered}
$$

then $P$ has a fixed point in $E_{0}$.

With these preparations, we can prove our main result in this paper.

Theorem 4.9. Let assumptions [H1], [H2], and [H3] hold. If the PC-mild solutions of Cauchy problem (3.16) are ultimate bounded, then system (3.15) has a $T_{0}$-periodic PC-mild solution.

Proof. By Theorem 3.5, (4.4) in Lemma 4.2, and Definition 4.5, the Cauchy problem (3.16) corresponding to the initial value $x(0)=\bar{x}$ has a $P C$-mild solution $x(\cdot, \bar{x})$ which is locally bounded. From ultimate boundedness and Lemma $4.7, x(\cdot, \bar{x})$ is bounded. Next, let $B>0$ be the bound in the definition of ultimate boundedness. Then by boundedness, there is a $B_{1}>B$ such that $\|\bar{x}\| \leq B$ implies $\|x(t, \bar{x})\| \leq B_{1}$ for $t \geq 0$. Furthermore, there is a $B_{2}>B_{1}$ such that $\|\bar{x}\| \leq B_{1}$ implies $\|x(t, \bar{x})\| \leq B_{2}$ for $t \geq 0$. Now, using ultimate boundedness again, there is a positive integer $m$ such that $\|\bar{x}\| \leq B_{1}$ implies $\|x(t, \bar{x})\| \leq B$ for $t \geq(m-2) T_{0}$. 
Define $y(\cdot, y(0))=x\left(\cdot+T_{0}, \bar{x}\right)$, then $y(0)=x\left(T_{0}, \bar{x}\right)=P(\bar{x})$. From (4.2) in Lemma 4.1, we obtain $P(y(0))=y\left(T_{0}, y(0)\right)=x\left(2 T_{0}, \bar{x}\right)$. Thus, $P^{2}(\bar{x})=P(P(\bar{x}))=P(y(0))=x\left(2 T_{0}, \bar{x}\right)$. Suppose there exists integer $m-1$ such that $P^{m-1}(\bar{x})=x\left((m-1) T_{0}, \bar{x}\right)$. By induction we arrive at

$$
P^{m}(\bar{x})=P^{m-1}(P(\bar{x}))=P^{m-1}(y(0))=y\left((m-1) T_{0}, y(0)\right)=x\left(m T_{0}, \bar{x}\right) .
$$

Thus, we obtain

$$
\begin{gathered}
\left\|P^{j-1}(\bar{x})\right\|=\left\|x\left((j-1) T_{0}, \bar{x}\right)\right\|<B_{2}, \quad j=1,2, \ldots, m-1,\|\bar{x}\|<B_{1} \\
\left\|P^{j-1}(\bar{x})\right\|=\left\|x\left((j-1) T_{0}, \bar{x}\right)\right\|<B, \quad j \geq m,\|\bar{x}\|<B_{1} .
\end{gathered}
$$

It comes from Lemma 4.3 that $P(\bar{x})=x\left(T_{0}, \bar{x}\right)$ on $X$ is compact. Now let

$$
\begin{gathered}
H=\left\{\bar{x} \in X:\|\bar{x}\|<B_{2}\right\}, \quad E_{2}=\text { cl. }(\operatorname{conv}(P(H))), \\
W=\left\{\bar{x} \in X:\|\bar{x}\|<B_{1}\right\}, \quad E_{1}=W \cap E_{2}, \\
G=\{\bar{x} \in X:\|\bar{x}\|<B\}, \quad E_{0}=\operatorname{cl} .(\operatorname{conv}(P(G))),
\end{gathered}
$$

where $\operatorname{conv}(Y)$ is the convex hull of the set $Y$ defined by $\operatorname{conv}(Y)=\left\{\sum_{i=1}^{n} \lambda_{i} y_{i} \mid n \geq 1, y_{i} \in\right.$ $\left.Y, \lambda_{i} \geq 0, \sum_{i=1}^{n} \lambda_{i}=1\right\}$, and cl. denotes the closure. Then we see that $E_{0} \subset E_{1} \subset E_{2}$ are convex subsets of $X$ with $E_{0}, E_{2}$ compact subsets and $E_{1}$ open relative to $E_{2}$, and from (4.16) one has

$$
\begin{gathered}
P^{j}\left(E_{1}\right) \subset P^{j}(W)=P P^{j-1}(W) \subset P(H) \subset E_{2}, \quad j=1,2, \ldots, m-1 ; \\
P^{j}\left(E_{1}\right) \subset P^{j}(W)=P P^{j-1}(W) \subset P(G) \subset E_{0}, \quad j=m, m+1, \ldots, 2 m-1 .
\end{gathered}
$$

We see that $P: E_{2} \rightarrow X$ is a continuous map continuous from Lemma 4.2. Consequently, from Horn's fixed point theorem, we know that the operator $P$ has a fixed point $x_{0} \in E_{0} \subset X$. By Lemma 4.1, we know that the $P C$-mild solution $x\left(\cdot, x_{0}\right)$ of the Cauchy problem (3.16) corresponding to the initial value $x(0)=x_{0}$ is just $T_{0}$-periodic. Therefore, $x\left(\cdot, x_{0}\right)$ is a $T_{0}$ periodic PC-mild solution of system (3.15). This proves the theorem.

At last, an example is given to illustrate our theory. Consider the following problem:

$$
\begin{array}{r}
\frac{\partial}{\partial t} x(t, y)=\Delta x(t, y)+x(t, y)+\int_{0}^{t}(1+\sin (t-s)) \sqrt{3 x^{2}(s, y)+2} d s, \\
y \in \Omega, \quad t \geq s, \quad t, s \in(0,2 \pi] \backslash\left\{\frac{1}{2} \pi, \pi, \frac{3}{2} \pi\right\},
\end{array}
$$


JinRong Wang et al.

$$
\begin{aligned}
\Delta x\left(\tau_{i}, y\right) & =x\left(\tau_{i}^{+}, y\right)-x\left(\tau_{i}^{-}, y\right)= \begin{cases}0.05 \operatorname{Ix}\left(\tau_{i}, y\right), & i=1, \\
-0.05 \operatorname{Ix}\left(\tau_{i}, y\right), & i=2, y \in \Omega, \tau_{i}=\frac{i}{2} \pi, i=1,2,3, \\
0.05 \operatorname{Ix}\left(\tau_{i}, y\right), & i=3,\end{cases} \\
\left.x(t, y)\right|_{y \in \partial \Omega} & =0, \quad t>0, \\
x(0, y) & =x(2 \pi, y),
\end{aligned}
$$

where $\Omega \subset \mathbb{R}^{3}$ is a bounded domain and $\partial \Omega \in C^{3}$.

Define $X=L_{2}(\Omega), D(A)=H^{2}(\Omega) \cap H_{0}^{1}(\Omega)$, and $A x=-\left(\partial^{2} x / \partial y_{1}^{2}+\partial^{2} x / \partial y_{2}^{2}+\partial^{2} x / \partial y_{3}^{2}\right)$ for $x \in D(A)$. Then, $A$ generates a compact semigroup $\{T(t), t \geq 0\}$ on $L_{2}(\Omega)$. Define $x(\cdot)(y)=x(\cdot, y), \sin (\cdot)(y)=\sin (\cdot, y), f\left(\cdot, x(\cdot), \int_{0}^{\cdot} g(\cdot, s, x) d s\right)(y)=x(\cdot)(y)+\int_{0}^{t}(1+\sin (\cdot-$ s)) $\sqrt{3 x^{2}(\cdot)+2} d s(y)$, and

$$
B_{i}= \begin{cases}0.05 I, & i=1, \\ -0.05 I, & i=2, \\ 0.05 I, & i=3 .\end{cases}
$$

Thus problem (4.19) can be rewritten as

$$
\begin{gathered}
\dot{x}(t)=A x(t)+f\left(t, x, \int_{0}^{t} g(t, s, x) d s\right), \quad t \geq s, t, s \in(0,2 \pi] \backslash\left\{\frac{1}{2} \pi, \pi, \frac{3}{2} \pi\right\}, \\
\Delta x\left(\frac{i}{2} \pi\right)=B_{i} x\left(\frac{i}{2} \pi\right), \quad i=1,2,3, \\
x(0)=x(2 \pi) .
\end{gathered}
$$

If the PC-mild solutions of Cauchy problem (4.21) are ultimate bounded, then all the assumptions in Theorem 4.9 are met, and our results can be used to system (4.19), that is, problem (4.19) has a $2 \pi$-periodic $P C$-mild solution $x_{2 \pi}(\cdot, y) \in P C_{2 \pi}\left([0+\infty) ; L_{2}(\Omega)\right)$, where

$$
P C_{2 \pi}\left([0,+\infty) ; L_{2}(\Omega)\right) \equiv\left\{x \in P C\left([0,+\infty) ; L_{2}(\Omega)\right) \mid x(t)=x(t+2 \pi), t \geq 0\right\} .
$$

\section{Acknowledgments}

This work is supported by Natural Science Foundation of Guizhou Province Education Department (no. 2007008) and Guizhou Province (no. 20052001). This work is also supported by the undergraduate carve out project of Department of Guiyang Science and Technology ([2008] no. 15-2).

\section{References}

[1] D. BaĬnov and P. Simeonov, Impulsive Differential Equations: Periodic Solutions and Applications, vol. 66 of Pitman Monographs and Surveys in Pure and Applied Mathematics, Longman Scientific \& Technical, Harlow, UK, 1993. 
[2] V. Lakshmikantham, D. D. Baĭnov, and P. S. Simeonov, Theory of Impulsive Differential Equations, vol. 6 of Series in Modern Applied Mathematics, World Scientific, Teaneck, NJ, USA, 1989.

[3] X. Liu, "Impulsive stabilization and applications to population growth models," The Rocky Mountain Journal of Mathematics, vol. 25, no. 1, pp. 381-395, 1995.

[4] T. Yang, Impulsive Control Theory, vol. 272 of Lecture Notes in Control and Information Sciences, Springer, Berlin, Germany, 2001.

[5] N. U. Ahmed, "Optimal impulse control for impulsive systems in Banach spaces," International Journal of Differential Equations and Applications, vol. 1, no. 1, pp. 37-52, 2000.

[6] N. U. Ahmed, "Some remarks on the dynamics of impulsive systems in Banach spaces," Dynamics of Continuous, Discrete \& Impulsive Systems. Series A, vol. 8, no. 2, pp. 261-274, 2001.

[7] N. U. Ahmed, K. L. Teo, and S. H. Hou, "Nonlinear impulsive systems on infinite dimensional spaces," Nonlinear Analysis: Theory, Methods E Applications, vol. 54, no. 5, pp. 907-925, 2003.

[8] N. U. Ahmed, "Existence of optimal controls for a general class of impulsive systems on Banach spaces," SIAM Journal on Control and Optimization, vol. 42, no. 2, pp. 669-685, 2003.

[9] W. Wei, X. Xiang, and Y. Peng, "Nonlinear impulsive integro-differential equations of mixed type and optimal controls," Optimization, vol. 55, no. 1-2, pp. 141-156, 2006.

[10] X. Xiang and W. Wei, "Mild solution for a class of nonlinear impulsive evolution inclusions on Banach space," Southeast Asian Bulletin of Mathematics, vol. 30, no. 2, pp. 367-376, 2006.

[11] X. Xiang, W. Wei, and Y. Jiang, "Strongly nonlinear impulsive system and necessary conditions of optimality," Dynamics of Continuous, Discrete E Impulsive Systems. Series A, vol. 12, no. 6, pp. 811-824, 2005.

[12] X. Yu, X. Xiang, and W. Wei, "Solution bundle for a class of impulsive differential inclusions on Banach spaces," Journal of Mathematical Analysis and Applications, vol. 327, no. 1, pp. 220-232, 2007.

[13] M. Benchohra, J. Henderson, and S. Ntouyas, Impulsive Differential Equations and Inclusions, vol. 2 of Contemporary Mathematics and Its Applications, Hindawi, New York, NY, USA, 2006.

[14] H. Amann, "Periodic solutions of semilinear parabolic equations," in Nonlinear Analysis: A Collection of Papers in Honor of Erich H. Rothe, pp. 1-29, Academic Press, New York, NY, USA, 1978.

[15] B. Aulbach and N. Van Minh, "Bounded and almost periodic solutions and evolution semigroups associated with nonautonomous functional differential equations," Abstract and Applied Analysis, vol. 5, no. 4, pp. 245-263, 2000.

[16] Y. S. Eidelman and I. V. Tikhonov, “On periodic solutions of abstract differential equations," Abstract and Applied Analysis, vol. 6, no. 8, pp. 489-499, 2001.

[17] J. Mawhin, "Periodic solutions of some semilinear wave equations and systems: a survey," Chaos, Solitons \& Fractals, vol. 5, no. 9, pp. 1651-1669, 1995.

[18] J. H. Liu, "Bounded and periodic solutions of differential equations in Banach space," Applied Mathematics and Computation, vol. 65, no. 1-3, pp. 141-150, 1994.

[19] J. H. Liu, "Bounded and periodic solutions of semilinear evolution equations," Dynamic Systems and Applications, vol. 4, no. 3, pp. 341-350, 1995.

[20] J. H. Liu, "Bounded and periodic solutions of finite delay evolution equations," Nonlinear Analysis: Theory, Methods $\mathcal{E}$ Applications, vol. 34, no. 1, pp. 101-111, 1998.

[21] J. H. Liu, T. Naito, and N. Van Minh, "Bounded and periodic solutions of infinite delay evolution equations," Journal of Mathematical Analysis and Applications, vol. 286, no. 2, pp. 705-712, 2003.

[22] D. Paşca, "Periodic solutions of a class of non-autonomous second-order differential inclusions systems," Abstract and Applied Analysis, vol. 6, no. 3, pp. 151-161, 2001.

[23] P. Sattayatham, S. Tangmanee, and W. Wei, "On periodic solutions of nonlinear evolution equations in Banach spaces," Journal of Mathematical Analysis and Applications, vol. 276, no. 1, pp. 98-108, 2002.

[24] X. Xiang and N. U. Ahmed, "Existence of periodic solutions of semilinear evolution equations with time lags," Nonlinear Analysis: Theory, Methods E Applications, vol. 18, no. 11, pp. 1063-1070, 1992.

[25] D. J. Guo and X. Liu, "Extremal solutions of nonlinear impulsive integrodifferential equations in Banach spaces," Journal of Mathematical Analysis and Applications, vol. 177, no. 2, pp. 538-552, 1993.

[26] J. R. Wang, "Linear impulsive periodic system on Banach space," in Proceedings of the 4th International Conference on Impulsive and Hybrid Dynamical Systems (ICIDSA '07), vol. 5, pp. 20-25, Nanning, China, July 2007. 
[27] J. R. Wang, X. Xiang, and W. Wei, "Linear impulsive periodic system with time-varying generating operators on Banach space," Advances in Difference Equations, vol. 2007, Article ID 26196, 16 pages, 2007.

[28] J. R. Wang, X. Xiang, and W. Wei, "Existence and global asymptotical stability of periodic solution for the $T$-periodic logistic system with time-varying generating operators and $T_{0}$-periodic impulsive perturbations on Banach spaces," Discrete Dynamics in Nature and Society, vol. 2008, Article ID 524945, 16 pages, 2008.

[29] N. U. Ahmed, Semigroup Theory with Applications to Systems and Control, vol. 246 of Pitman Research Notes in Mathematics Series, Longman Scientific \& Technical, Harlow, UK, 1991. 


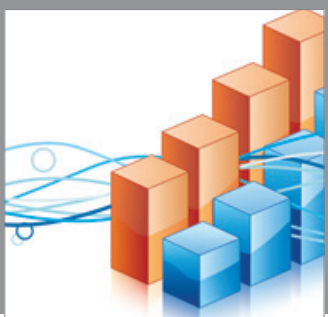

Advances in

Operations Research

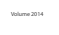

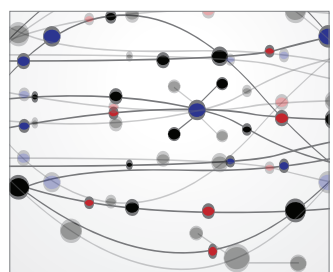

\section{The Scientific} World Journal
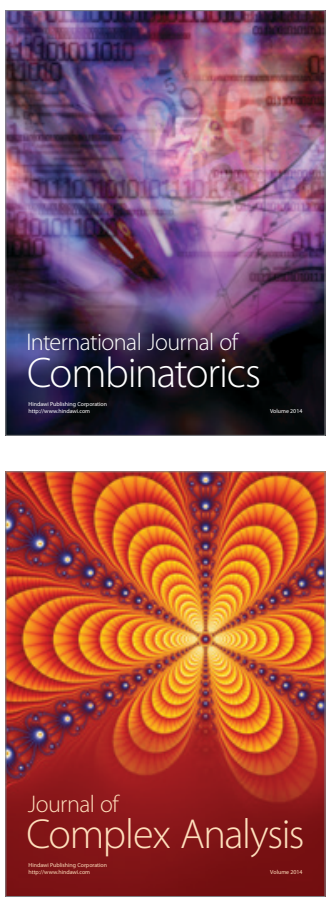

International Journal of

Mathematics and

Mathematical

Sciences
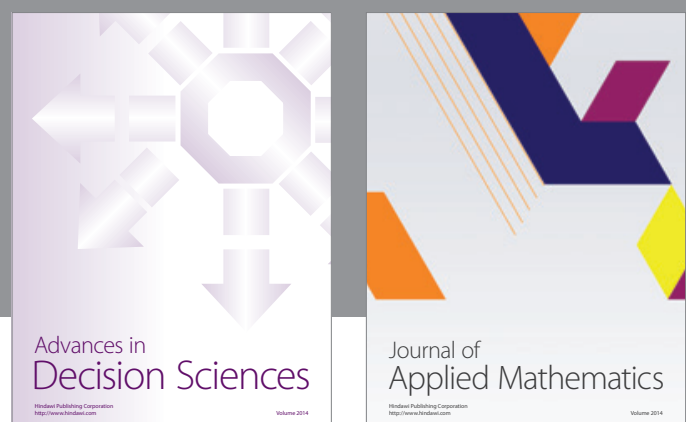

Journal of

Applied Mathematics
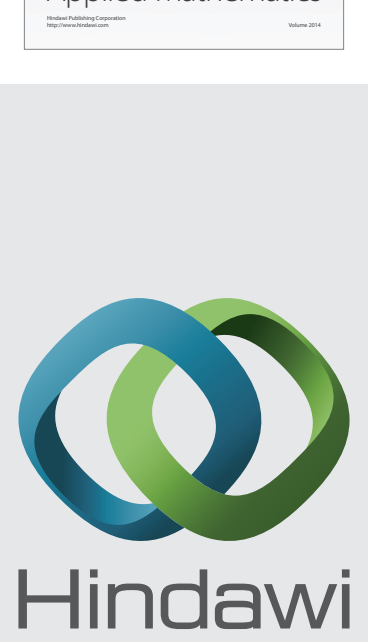

Submit your manuscripts at http://www.hindawi.com
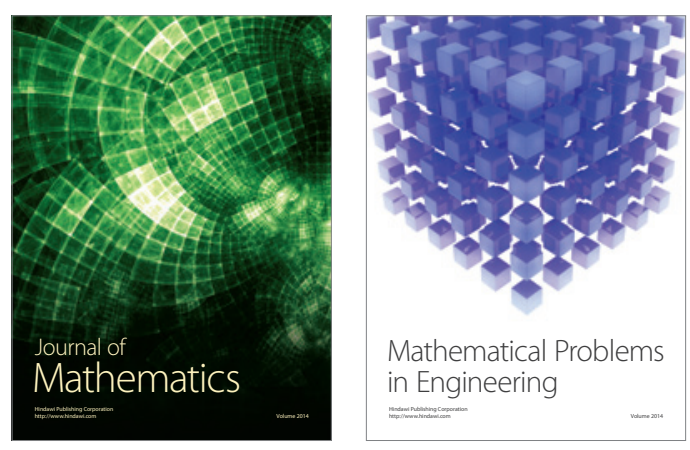

Mathematical Problems in Engineering
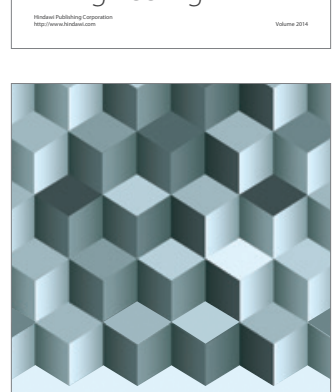

Journal of

Function Spaces
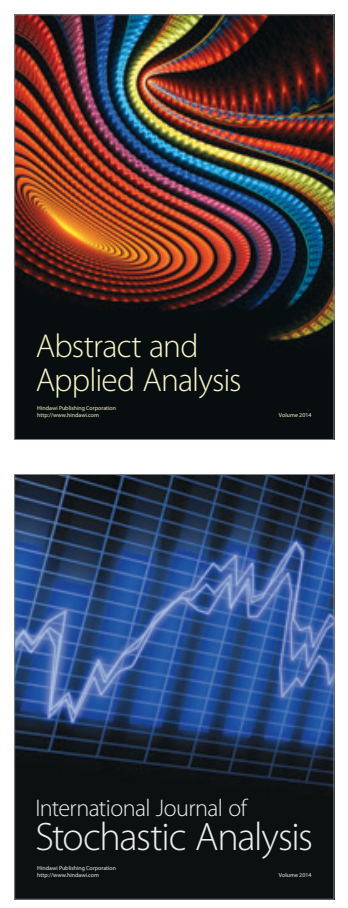

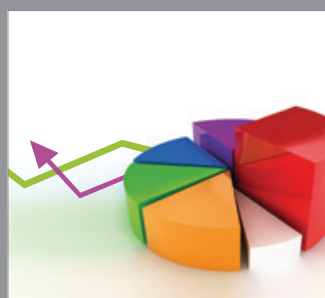

ournal of

Probability and Statistics

Promensencen
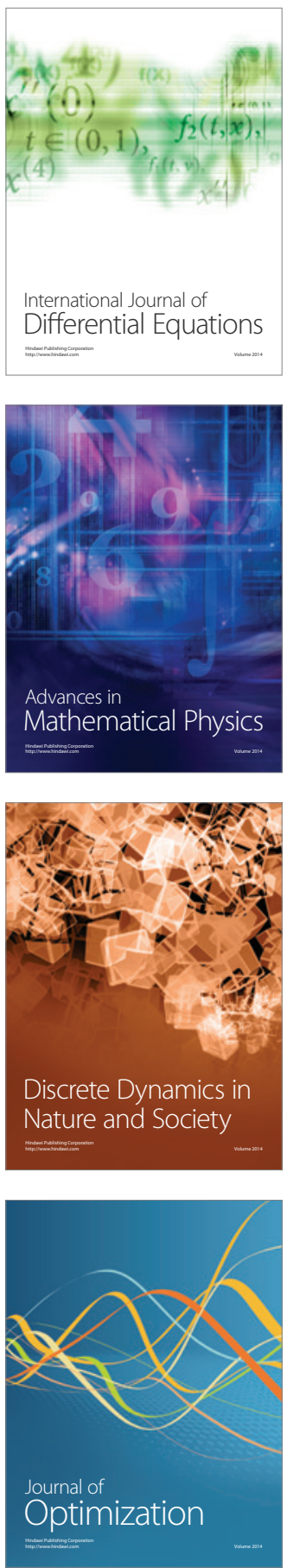